\title{
Large Orbital Magnetic Moment and Strong Perpendicular Magnetic Anisotropy in Heavily Intercalated $\mathrm{Fe}_{x} \mathrm{TiS}_{2}$
}

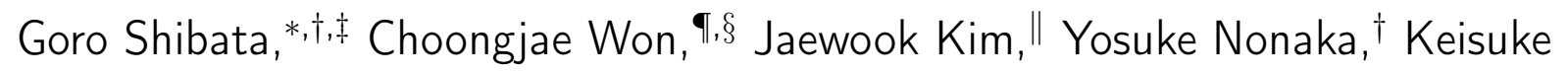 \\ Ikeda, ${ }^{\dagger}$ Yuxuan Wan, ${ }^{\dagger, \perp}$ Masahiro Suzuki, ${ }^{\dagger}$ Tsuneharu Koide, ${ }^{\#}$ Arata Tanaka, ${ }^{\circledR}$ \\ Sang-Wook Cheong, $\|, \uparrow, \S$ and Atsushi Fujimori, ${ }^{*}, \Delta$ \\ $\dagger$ Department of Physics, The University of Tokyo, Tokyo, Japan \\ $\ddagger$ Department of Applied Physics, Tokyo University of Science, Tokyo, Japan \\ \Max Planck POSTECH/Korea Research Initiative, Pohang University of Science and \\ Technology, Pohang, Korea \\ $\S$ Laboratory of Pohang Emergent Materials, Pohang Accelerator Laboratory, Pohang, Korea \\ ||Department of Physics and Astronomy, Rutgers University, Piscataway, New Jersey, USA \\ $\perp$ The Institute for Solid State Physics, The University of Tokyo, Kashiwa, Japan \\ \#Institute of Materials Structure Science, High Energy Accelerator Research Organization, \\ Tsukuba, Ibaraki, Japan \\ @Department of Quantum Matter, Hiroshima University, Hiroshima, Japan \\ $\triangle$ Department of Applied Physics, Waseda University, Tokyo, Japan \\ E-mail: shibata@rs.tus.ac.jp; fujimori@phys.s.u-tokyo.ac.jp
}

Phone: +81(3)-5876-1717 


\begin{abstract}
Titanium disulfide $\mathrm{TiS}_{2}$, which is a member of layered transition-metal dichalcogenides with the $1 \mathrm{~T}-\mathrm{CdI}_{2}$-type crystal structure, is known to exhibit a wide variety of magnetism through intercalating various kinds of transition-metal atoms of different concentrations. Among them, Fe-intercalated titanium disulfide $\mathrm{Fe}_{x} \mathrm{TiS}_{2}$ is known to be ferromagnetic with strong perpendicular magnetic anisotropy (PMA) and large coercive fields $\left(H_{\mathrm{c}}\right)$. In order to study the microscopic origin of the magnetism of this compound, we have performed X-ray absorption spectroscopy (XAS) and X-ray magnetic circular dichroism (XMCD) measurements on single crystals of heavily intercalated $\mathrm{Fe}_{x} \mathrm{TiS}_{2}(x \sim 0.5)$. The grown single crystals showed a strong PMA with a large $H_{\mathrm{c}}$ of $\mu_{0} H_{\mathrm{c}} \simeq 1.0 \mathrm{~T}$. XAS and XMCD spectra showed that Fe is fully in the valence states of $2+$ and that $\mathrm{Ti}$ is in an itinerant electronic state, indicating electron transfer from the intercalated $\mathrm{Fe}$ atoms to the host $\mathrm{TiS}_{2}$ bands. The $\mathrm{Fe}^{2+}$ ions were shown to have a large orbital magnetic moment of $\simeq 0.59 \mu_{\mathrm{B}} / \mathrm{Fe}$, to which, combined with the spin-orbit interaction and the trigonal crystal field, we attribute the strong magnetic anisotropy of $\mathrm{Fe}_{x} \mathrm{TiS}_{2}$.
\end{abstract}

\title{
Introduction
}

Ferromagnetism in systems where the distance between $3 d$ transition-metal atoms is unusually long has attracted strong interest of researchers. Typical systems are diluted ferromagnetic semiconductors such as $\mathrm{Ga}_{1-x} \mathrm{Mn}_{x} \mathrm{As}^{\underline{1}}$ and double perovskites such as $\mathrm{Sr}_{2} \mathrm{FeMoO}_{6}, \underline{\underline{2}}$ where hybridization between the relatively localized $3 d$ orbitals of the transition-metal atoms and the itinerant, more extended orbitals plays the crucial role.

Recently, there has been keen interest in transition-metal dichalcogenides (TMDs) as a family of two-dimensional materials due to their novel physical properties. $\underline{\underline{3}}^{-6}$ TMDs have stacked crystal structures in which two-dimensional $\mathrm{AX}_{2}$ layers consisting of triangular lattices of the transition-metal atoms $\mathrm{A}$ sandwiched by the chalcogen atoms $\mathrm{X}$ are coupled 
with each other through weak van der Waals forces. In these compounds, one can intercalate other $3 d$ transition-metal atoms $\mathrm{M}$ into the van der Waals gaps. The intercalated compounds, $\mathrm{M}_{x} \mathrm{AX}_{2}$, are known to exhibit a wide variety of structural, magnetic, and transport properties, including paramagnetic, ferromagnetic (FM), antiferromagnetic (AFM), and spin-glass behaviors and magneto-transport, depending on the host compound, the intercalated atom M, and its concentration $x .^{\underline{-10}}$ Titanium disulfide $\mathrm{TiS}_{2}$ is one of the TMDs with the $1 \mathrm{~T}-\mathrm{CdI}_{2}$-type layered crystal structure, where the $\mathrm{Ti}$ atom is surrounded by six $\mathrm{S}$ atoms octahedrally. Extensive studies on the structural, $\underline{11}$ transport, $, 12,13$ and magnetic properties ${ }^{9,10,14}$ of $\mathrm{M}_{x} \mathrm{TiS}_{2}$ have been made so far. Among the transition-metal intercalated titanium disulfides, the Fe-intercalated compound $\mathrm{Fe}_{x} \mathrm{TiS}_{2}$ has been found to be $\mathrm{FM}$ in an exceptionally wide range of Fe concentrations $x(0.2 \lesssim x \leq 1), \underline{9,10,14-16}$ Moreover, the FM $\mathrm{Fe}_{x} \mathrm{TiS}_{2}$ has been shown to exhibit strong perpendicular magnetic anisotropy (PMA) with a large coercive field $\left(H_{\mathrm{c}}\right)$ of $\gtrsim 1 \mathrm{~T} \cdot \underline{9,10,14}-\underline{16}$ It has also been reported that this FM state exhibits spin-glass-like behaviors, called the "cluster-glass" state, observed by ac susceptibility measurements, $\underline{9,16}$ magnetotransport measurements,,$\underline{17}$ and neutron scattering. $\underline{18}$ Effects of the ordering of the intercalated Fe atoms, observed by transmission electron microscopy, $, \underline{17}, 19$ are also thought to be important in order to explain the $x$ dependence of the magnetic phases.

In an attempt to understand the origin of the diverse magnetic properties of these intercalated compounds $\mathrm{M}_{x} \mathrm{TiS}_{2}$ from the viewpoint of electronic structures, first-principles calculations $^{20}-22$ and photoemission spectroscopy $\stackrel{22-28}{2}$ studies have been performed so far. The importance of hybridization between the guest transition-metal atoms and the host $\mathrm{TiS}_{2}$ has been pointed out based on the first-principles calculations. $\underline{20}-22$ Magnetic coupling between the guest transition-metal atoms thus deduced is mapped onto the Ruderman-Kittel-KasuyaYosida (RKKY) model, and is used to explain the complex magnetic phases as a function of the concentration $x . \underline{29}, 30$ As for the magnetism of $\mathrm{Fe}_{x} \mathrm{TiS}_{2}, \mathrm{X}$-ray absorption spectroscopy (XAS) and X-ray magnetic circular dichroism (XMCD) measurements for $x=0-0.33$ have 
also been performed. $\frac{27}{2}$ Systematic changes in the spectral line shapes as functions of $x$ for both the $\mathrm{Fe}$ and the $\mathrm{Ti}$ absorption edges have been observed. 27 The presence of a large orbital magnetic moment of Fe observed by XMCD has also been pointed out. ${ }^{27}$ However, the relationship between the observed large orbital magnetic moment and the strong PMA has not been discussed in sufficient detail. In addition, the magnetism of $\mathrm{Fe}_{x} \mathrm{TiS}_{2}$ at higher $x$ 's is more interesting because it has higher FM transition temperatures and coercive fields than that at lower $x$ 's. $\underline{9}, \underline{10}$ Therefore, spectroscopic studies on heavily intercalated $\mathrm{Fe}_{x} \mathrm{TiS}_{2}$ crystals have been desired.

In the present study, we have performed XAS and XMCD experiments on single crystals of heavily intercalated $\mathrm{Fe}_{x} \mathrm{TiS}_{2}(x \sim 0.5)$. The grown single crystals showed a strong PMA with a large $H_{\mathrm{c}}$ of $\mu_{0} H_{\mathrm{c}} \simeq 1.0 \mathrm{~T}$. XAS and XMCD spectra indicate electron transfer from the intercalated Fe atoms to the $\mathrm{TiS}_{2}$ host. The $\mathrm{Fe}^{2+}$ ions are shown to have a large orbital magnetic moment of $\simeq 0.59 \pm 0.08 \mu_{\mathrm{B}} / \mathrm{Fe}$, which would be associated with the strong magnetic anisotropy of $\mathrm{Fe}_{x} \mathrm{TiS}_{2}$.

\section{Methods}

$\mathrm{Fe}_{x} \mathrm{TiS}_{2}$ single crystals with $x \sim 0.5$ were grown by the typical chemical vapor deposition method. First, stoichiometric mixed powder with an $\mathrm{I}_{2}$ transport agent in evacuated quartz tubes was heated at $500 \mathrm{~K}$ for $24 \mathrm{~h}$ to prevent the explosion of the ampule. Then, the sealed ampule was heated at $1173 \mathrm{~K}$ for 2 weeks with a temperature difference of $50 \mathrm{~K}$ between the both ends of the ampule. Detailed methods for the sample preparation are described

in Ref. 31. The crystallinity of the sample was checked by X-ray diffraction (XRD) using the X'Pert Pro diffractometer from PANalytical. The conventional $\theta-2 \theta$ method in the specular geometry was adopted. The X-ray source was the $\mathrm{Cu} K \alpha$ line (wavelength $\lambda=$ $0.15418 \mathrm{~nm}$ ) and the operation voltage and current of the X-ray tube were $40 \mathrm{kV}$ and $30 \mathrm{~mA}$, respectively. The stoichiometry of the crystals was checked by energy dispersive X-ray (EDX) 
spectroscopy equipped with a scanning electron microscope (SEM). The magnetic properties of the grown crystals were measured using the superconducting quantum interference device magnetometry.

The XAS and XMCD spectra at the Fe $L_{2,3}(2 p \rightarrow 3 d)$ and Ti $L_{2,3}$ edges were measured using a vector-magnet XMCD measurement apparatus ${ }^{32,33}$ installed at beamline BL-16A of Photon Factory, High Energy Accelerator Research Organization (KEK-PF). The samples were cleaved in situ prior to measurements. Figure 1 schematically describes the experimental geometry of the XMCD measurements. The vacuum was maintained at $\sim 1 \times 10^{-7}$ $\mathrm{Pa}$ during the entirety of the measurements. The samples were cooled down to $T=30 \mathrm{~K}$ without a magnetic field. The magnetic field was then applied parallel to the $c$-axis of the samples with the X-ray incident angle of $45^{\circ}$. The maximum magnetic field was $1 \mathrm{~T}$. XAS signals were collected in the total electron yield mode.

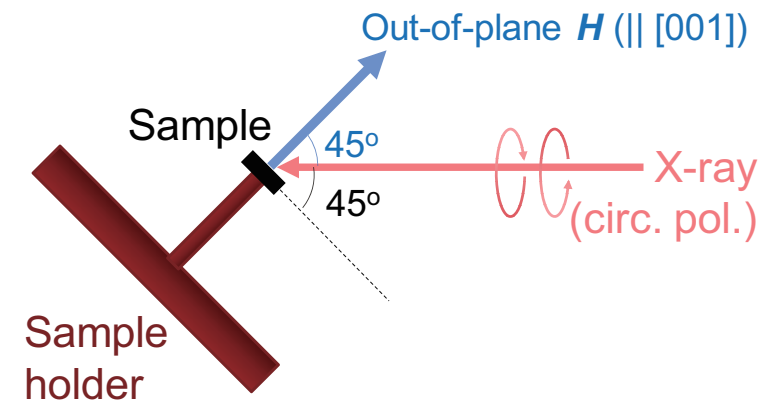

Figure 1: Experimental geometry of the X-ray magnetic circular dichroism (XMCD) measurements.

From the measured XAS and XMCD spectra, the orbital $\left(M_{\text {orb }}\right)$ and spin $\left(M_{\text {spin }}\right)$ magnetic moments of Fe were deduced using the XMCD sum rules. $\underline{34}, \underline{35}$ The explicit forms of the XMCD sume rules are given as follows: $\underline{34}, 35$

$$
\begin{aligned}
M_{\mathrm{orb}} & =-\frac{4}{3} \frac{\int_{L_{3}+L_{2}}\left(\mu_{+}(E)-\mu_{-}(E)\right) d E}{\int_{L_{3}+L_{2}}\left(\mu_{+}(E)+\mu_{-}(E)\right) d E}\left(10-N_{d}\right), \\
M_{\text {spin }} & =-\frac{2 \int_{L_{3}}\left(\mu_{+}(E)-\mu_{-}(E)\right) d E-4 \int_{L_{2}}\left(\mu_{+}(E)-\mu_{-}(E)\right) d E}{\int_{L_{3}+L_{2}}\left(\mu_{+}(E)+\mu_{-}(E)\right) d E}\left(10-N_{d}\right),
\end{aligned}
$$


where $\mu_{+}(E)$ and $\mu_{-}(E)$ are the XAS spectra for the right- and left-circular polarizations, respectively, $L_{3}\left(L_{2}\right)$ are the absorption peaks corresponding to the Fe $2 p_{3 / 2} \rightarrow 3 d\left(2 p_{1 / 2} \rightarrow\right.$ $3 d$ ) transition, $E$ is the photon energy, $N_{d}$ is the number of electrons in the transitionmetal $3 d$ band, and $M_{\text {orb }}$ and $M_{\text {spin }}$ are given in the unit of measure of $\mu_{\mathrm{B}} /$ atom. $N_{d}=$ 6, which is the nominal number of electrons in an $\mathrm{Fe}^{2+}$ ion, was assumed in the present study. In the original references, $\underline{34,35}$ the denominator is the sum of three XAS spectra, $\left(\mu_{+}(E)+\mu_{-}(E)+\mu_{0}(E)\right)$ (where $\mu_{0}(E)$ is the XAS spectra for the linear polarization along the X-ray incident direction), which were approximated by $(3 / 2)\left(\mu_{+}(E)+\mu_{-}(E)\right)$ here. In order to apply the sum rules to measured spectra, a continuum background was subtracted from the raw XAS spectra as follows: First, a polygonal line bent at the $L_{3}$ XAS peak position was subtracted so that the pre-edge and the post-edge regions became horizontal. Then, a smoothed two-step background was subtracted, which is composed of two arctangent functions with relative heights of $2: 1$ centered at the peak positions of the $L_{3}$ and $L_{2}$ edges. We note that the arbitrariness of these background subtraction procedures causes systematic errors of $\sim \pm(10-15) \%$ in the integrated XAS intensities. In Eq. (2), the spectra have been divided into the $L_{3^{-}}$and $L_{2}$-edge regions at $E=718 \mathrm{eV}$. It is known that, when using the sum rules, the division of the XAS and XMCD spectra into the $L_{3}$ and $L_{2}$ edges at a certain energy may result in the underestimation of $M_{\text {spin }} \cdot \underline{36}, 37$ The presence of an additional term called the magnetic dipole term $M_{T}, \stackrel{34,38,39}{,}$ which has been omitted in Eq. (2), may also cause some systematic errors in $M_{\text {spin }}$. According to Ref. 37, the magnitude of the systematic errors of $M_{\text {spin }}$ due to the incomplete $L_{3}-L_{2}$ separation and the magnetic dipole term $M_{T}$ are estimated to be about $-10 \%$ and $\pm 5 \%$, respectively, in the case of an $\mathrm{Fe}^{2+}$ ion. Hereafter, only the statistical errors of $M_{\text {orb }}$ and $M_{\text {spin }}$ are presented.

The spectral line shapes of the XAS and XMCD spectra were analyzed based on the configuration-interaction (CI) cluster model $\underline{40}$ using the XTLS 8.5 package. $\stackrel{40}{ }$ We assumed an $\left[\mathrm{FeS}_{6}\right]^{10-}$ cluster (i.e., divalent $\mathrm{Fe}$ ) with $D_{3 d}$ symmetry, i.e., the $\mathrm{FeS}_{6}$ octahedron is slightly elongated or shrunk along a trigonal axis. The following parameters were adjusted in order 
to obtain the best-fit spectra: $U_{d d}$ (Coulomb energy between two Fe $3 d$ valence electrons), $U_{d c}$ (Coulomb energy between the Fe $3 d$ electron and the Fe $2 p$ core electron), $\Delta$ (chargetransfer energy), $(p d \sigma)$ (Slater-Koster parameters), 10Dq (octahedral crystal field), $D_{\operatorname{trg}}$ (trigonal crystal field), and $H_{\text {mol }}$ (molecular field). Spin-orbit interaction (SOI) and the Slater integrals were estimated from atomic Hartree-Fock calculations. For the Slater integrals, $80 \%$ of the values deduced from the atomic Hartree-Fock calculation were used. In the CI calculation, the ground state was assumed to be a linear combination of the $3 d^{6}, 3 d^{7} \underline{L}$, and $3 d^{8} \underline{L}^{2}$ configurations, where $\underline{L}$ is a ligand hole.

\section{Results and Discussion}

\section{Sample Characterization}

Figure 2 shows the XRD profile of an $\mathrm{Fe}_{x} \mathrm{TiS}_{2}(x \sim 0.5)$ single crystal. Sharp Bragg peaks along the [001] direction are clearly observed, confirming the layered structure of the grown crystal. From the obtained XRD profile, the out-of-plane lattice parameter $c$ has been estimated to be $0.572 \mathrm{~nm}$, close to the value $c=0.574 \mathrm{~nm}$ obtained in the previous study for $\mathrm{Fe}_{x} \mathrm{TiS}_{2}$ with $x=0.5 . \underline{\underline{11}}$

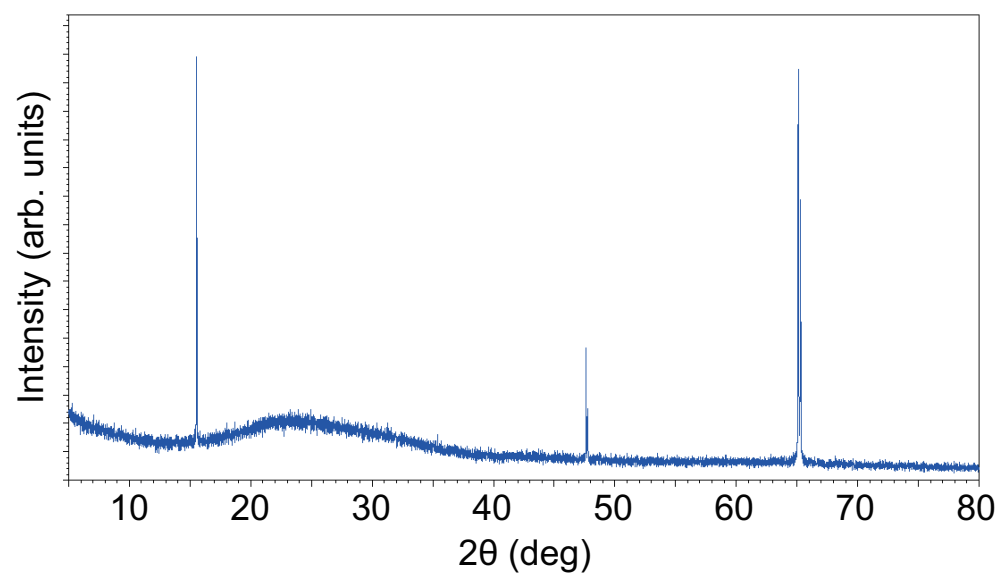

Figure 2: X-ray diffraction (XRD) profile along the out-of-plane [001] direction of an $\mathrm{Fe}_{x} \mathrm{TiS}_{2}$ $(x \sim 0.5)$ single crystal.

Figure 3(a) shows the SEM image of the $\mathrm{Fe}_{x} \mathrm{TiS}_{2}$ single crystal on a carbon tape. The 
typical sample diameter is $0.5-1 \mathrm{~mm}$. Regions with flat surfaces can be seen with typical sizes of a few hundred micrometers squared. The EDX spectrum of the crystal is shown in Fig. 3(b). From the spectral intensities, the atomic ratio of each element has been estimated to be $\mathrm{Fe}: \mathrm{Ti}: \mathrm{S}=12.7: 28.5: 58.8$, which corresponds to the chemical formula $\mathrm{Fe}_{0.432} \mathrm{Ti}_{0.968} \mathrm{~S}_{2}$. This confirms that more Fe atoms are intercalated in the present $\mathrm{Fe}_{x} \mathrm{TiS}_{2}$ crystal than those in the previous XMCD study $(x \leq 0.33), \underline{27}$ although the Fe concentration is slightly lower than the nominal value $(x=0.5)$.
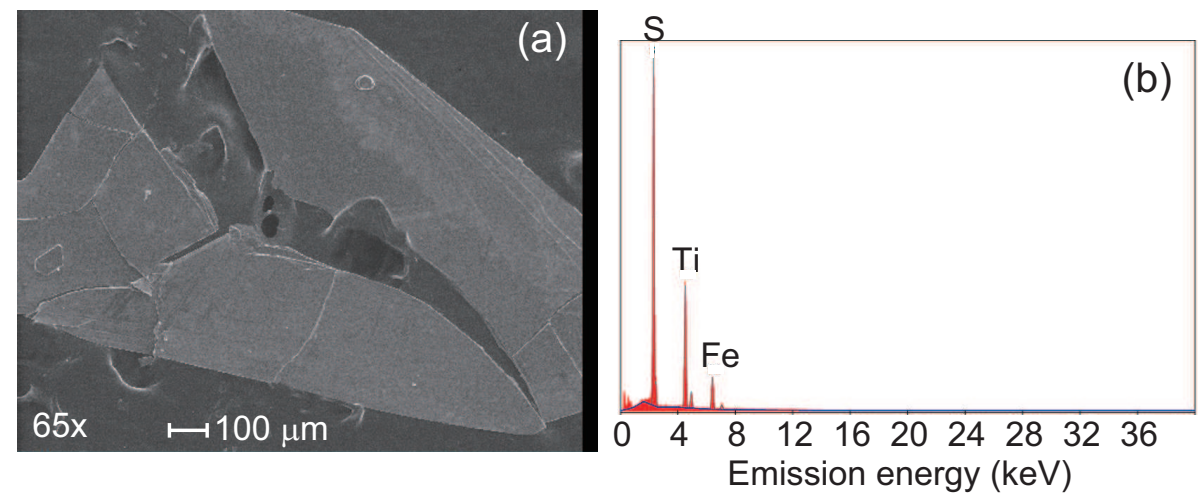

Figure 3: Energy dispersive X-ray spectroscopy (EDX) analysis of an $\mathrm{Fe}_{x} \mathrm{TiS}_{2}$ single crystal. (a) Scanning electron microscope (SEM) image of the $\mathrm{Fe}_{x} \mathrm{TiS}_{2}$ single crystal on a carbon tape. (b) EDX spectrum.

From the temperature dependence of the magnetization, the Curie temperature of the crystal has been estimated to be $\sim 70 \mathrm{~K}$. Figure 4 shows the zero-field cooled (ZFC) magnetization curve of the $\mathrm{Fe}_{x} \mathrm{TiS}_{2}(x \sim 0.5)$ single crystal measured at $2 \mathrm{~K}$ with an applied magnetic field $\boldsymbol{H}$ parallel to the $c$-axis (trigonal axis) up to $\mu_{0}|\boldsymbol{H}|=7 \mathrm{~T}$. In the initial magnetization process, a sudden jump in the magnetization $M$ is observed around $\mu_{0} H \sim$ $1 \mathrm{~T}$, suggesting a transition from the AFM to FM states. After magnetic saturation, a clear rectangular hysteresis loop is observed with a coercive field $H_{\mathrm{c}}$ of $\mu_{0} H_{\mathrm{c}} \sim 1 \mathrm{~T}$. The obtained $H_{\mathrm{c}}$ is similar to those of polycrystalline $\mathrm{Fe}_{x} \mathrm{TiS}_{2}$ with $(x=0.33-0.45)$ and single-crystalline $\mathrm{Fe}_{0.33} \mathrm{TiS}_{2}$ reported in the previous study. 10 The large hysteresis loop suggests that the crystal exhibits a strong PMA with the magnetic easy axis along the $c$-axis. We note that quantitative estimate of the saturation magnetization $M_{\text {sat }}$ from the raw magnetization curve 
is difficult due to a large uncertainty of the sample mass. We have, therefore, plotted the magnetization curve normalized by $M_{\text {sat }}$ in Fig. 4. We shall deduce the values of $M_{\text {sat }}$ and the uniaxial magnetic anisotropy energy (MAE) $K_{\mathrm{u}}$ by the XMCD measurements afterward.

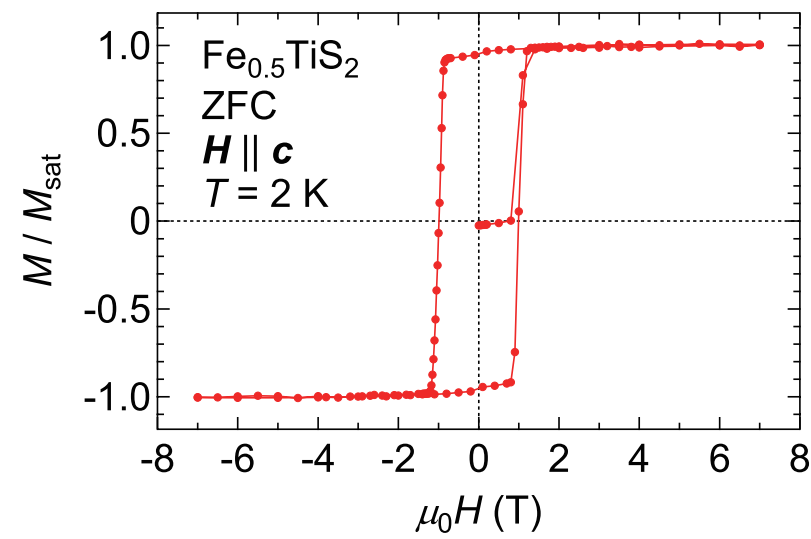

Figure 4: Normalized magnetization curve of the $\mathrm{Fe}_{0.5} \mathrm{TiS}_{2}$ single crystal.

\section{X-ray Magnetic Circular Dichroism Spectroscopy}

Figure 5(a) shows the helicity-averaged XAS spectra of the $\mathrm{Fe}_{x} \mathrm{TiS}_{2}(x \sim 0.5)$ single crystal at the Fe $L_{2,3}\left(2 p_{1 / 2,3 / 2} \rightarrow 3 d\right)$ absorption edges. The XAS spectra of divalent and trivalent iron oxides and metallic iron are also shown as references. $\cdot \underline{41}, \underline{42}$ The overall spectral line shape of $\mathrm{Fe}_{0.5} \mathrm{TiS}_{2}$ is similar to that of $\mathrm{FeO}$. Especially, the small shoulder around $h \nu=$ $706.5 \mathrm{eV}$ is characteristic of divalent $\mathrm{Fe}$, showing that the iron atom is essentially in the $\mathrm{Fe}^{2+}$ valence state. Reflecting the large hybridization strength of sulfides and high electrical conductivity compared to oxides, the XAS spectrum of $\mathrm{Fe}_{0.5} \mathrm{TiS}_{2}$ shows broader absorption peaks and more extended high-energy tails than that of FeO. One may think that the XAS spectrum of $\mathrm{Fe}_{0.5} \mathrm{TiS}_{2}$ is also similar to that of the Fe metal. As indicated in Fig. 5(a) by arrows, however, the XAS spectra of $\mathrm{Fe}_{0.5} \mathrm{TiS}_{2}$ and $\mathrm{FeO}$ have several multiplet structures in common, while they are absent in the XAS spectrum of metallic Fe reflecting its itinerant electronic structures. This indicates that the amount of possible metallic iron clusters, if any, is small in the present $\mathrm{Fe}_{0.5} \mathrm{TiS}_{2}$ crystal. The difference of the peak positions of the multiplet structures between $\mathrm{Fe}_{0.5} \mathrm{TiS}_{2}$ and $\mathrm{FeO}$ is probably due to the different hybridization strength 
and crystal-field splitting between oxides and sulfides.

In Fig. 5(b), the helicity-averaged XAS spectra of the $\mathrm{Fe}_{x} \mathrm{TiS}_{2}(x \sim 0.5)$ single crystal at the $\mathrm{Ti} L_{2,3}$ absorption edges are compared with those of $\mathrm{Fe}_{x} \mathrm{TiS}_{2}(0 \leq x \leq 0.33)^{27,43}$ and titanium oxides. $\underline{44}$ The spectral line shape of the present $\mathrm{Fe}_{0.5} \mathrm{TiS}_{2}$ crystal is close to those of $\mathrm{Fe}_{x} \mathrm{TiS}_{2}$ with $x \leq 0.33 .{ }^{27}$ One can see that, as $x$ increases, the absorption peaks become broader and the energy splitting between the two principal peaks in each of the $L_{3}$ and $L_{2}$ edges become systematically smaller. The shoulder structures around $h \nu=457$ and $463 \mathrm{eV}$ in the present XAS spectra are more diffuse than those for $x=0.33$, confirming that a larger amount of Fe atoms are intercalated in the present sample than in the previous ones. These spectral changes with increasing $x$ are considered to be due to electron doping from the intercalated Fe atoms into the $\mathrm{TiS}_{2}$ host. As can be seen from the figure, the spectral line shapes of $\mathrm{Fe}_{x} \mathrm{TiS}_{2}$ with $x \neq 0$ cannot be described as a superposition of the $\mathrm{Ti}^{4+}$ and $\mathrm{Ti}^{3+}$ valence states. This implies that the electrons doped from Fe into $\mathrm{TiS}_{2}$ do not lead to a mixed-valence state consisting of the $\mathrm{Ti}^{4+}$ and $\mathrm{Ti}^{3+}$ ionic states, but have rather itinerant character.

Figures 5 (c)-5(f) show the helicity-dependent XAS spectra and the XMCD spectra at the Fe $L_{2,3}$ and Ti $L_{2,3}$ edges. The XMCD spectra in Figs. 55(e) and 5)(f) clearly show that not only Fe but also Ti exhibits finite XMCD signals. This suggests that the observed ferromagnetism is not due to magnetic impurities such as metallic iron or iron compounds but due to the intrinsic properties of the $\mathrm{Fe}_{0.5} \mathrm{TiS}_{2}$ crystal. First-principle calculations ${ }^{20,21}$ show that the band structure of the transition-metal-intercalated $\mathrm{TiS}_{2}$ is significantly modified from that of the nonintercalated $\mathrm{TiS}_{2}$ through hybridization between the intercalated atoms and the host compound. In the case of Fe intercalation, the down-spin Fe $3 d$ states hybridize with the Ti $3 d$ states to form spin-polarized bands near the Fermi level. $\underline{20}$ As a result, Ti exhibits small but finite spin polarization. $\underline{20}$ The present XMCD result is qualitatively consistent with this calculation.

Using the XMCD sum rules, $\underline{34}, \underline{35}$ we have deduced the spin $\left(M_{\text {spin }}\right)$ and orbital magnetic 

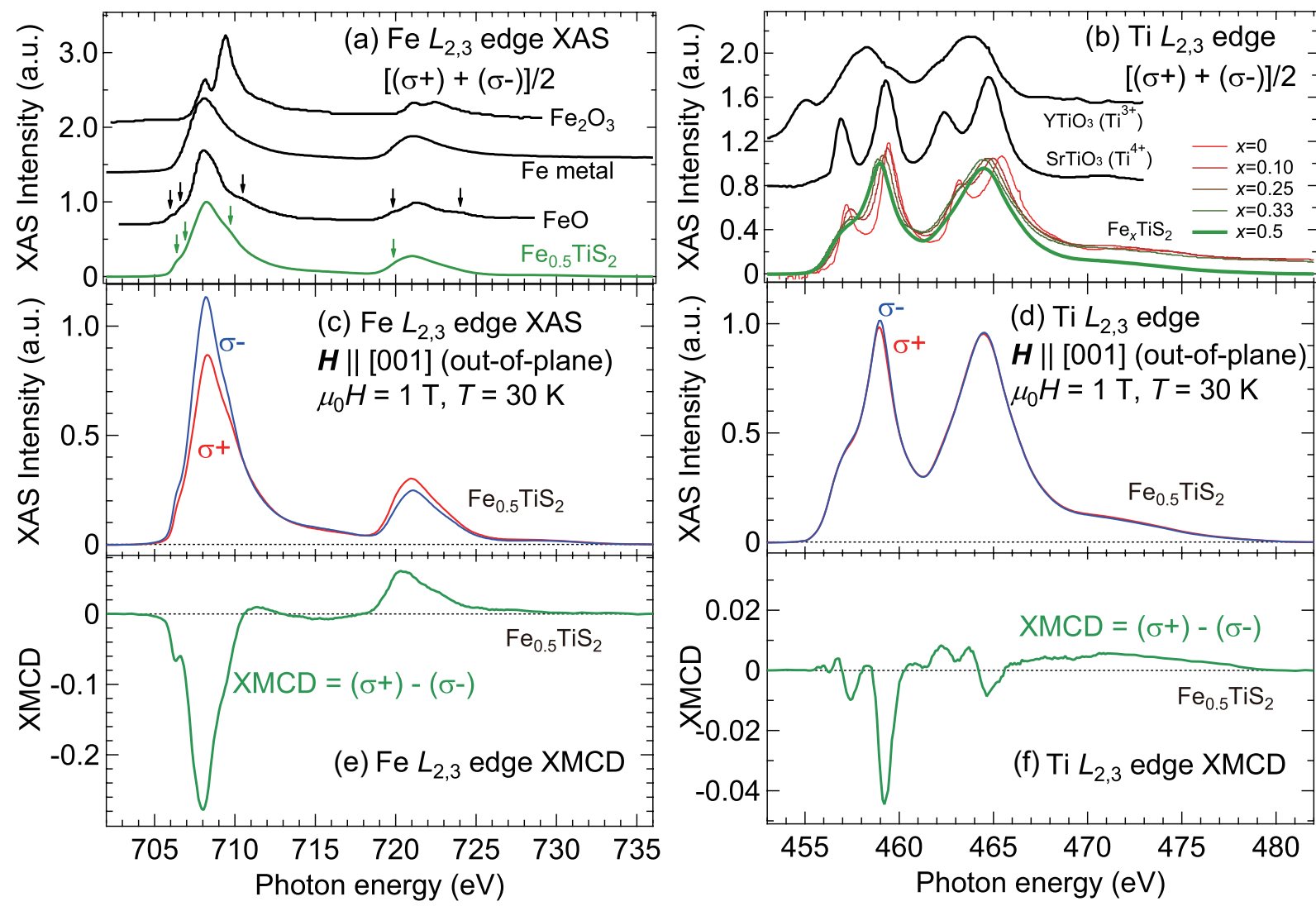

Figure 5: X-ray absorption spectroscopy (XAS) and XMCD spectra. (a,b) Helicity-averaged XAS spectrum of $\mathrm{Fe}_{0.5} \mathrm{TiS}_{2}$ at the Fe $L_{2,3}\left(2 p_{1 / 2,3 / 2} \rightarrow 3 d\right)$ (a) and $\mathrm{Ti} L_{2,3}$ (b) absorption edges. The reference XAS spectra of iron oxides, metallic iron, and titanium oxides are taken from Refs. 41 , 42, and 44, respectively. The Ti XAS spectra of $\mathrm{TiS}_{2}$ and $\mathrm{Fe}_{x} \mathrm{TiS}_{2}$ $(0 \leq x \leq 0.33)$ are taken from Refs. 43 and 27, respectively. Arrows in Panel (a) indicate the multiplet structures of $\mathrm{Fe}^{2+}$, which are absent in the metallic Fe. (c,d) Helicity-dependent XAS spectra of the $\mathrm{Fe}_{0.5} \mathrm{TiS}_{2}$ single crystal at the Fe $L_{2,3}$ (c) and $\mathrm{Ti} L_{2,3}$ (d) edges. (e,f) XMCD spectra at the Fe $L_{2,3}$ (e) and Ti $L_{2,3}$ (f) edges. The spectra were measured at the temperature $(T)$ of $30 \mathrm{~K}$ under the magnetic field $\left(\mu_{0} H\right)$ of $1 \mathrm{~T}$ applied parallel to the $c$-axis ([001] axis). The spectra have been normalized so that the peak height of the helicity-averaged XAS spectra at the $L_{3}$ edge is equal to unity. In Panels (a)-(d), two-step backgrounds has been subtracted from the raw XAS spectra. 
moments $\left(M_{\mathrm{orb}}\right)$ of Fe. We note that the $M_{\text {spin }}$ of Ti cannot be deduced from the spin sum rule due to the too small spin-orbit splitting of the Ti $2 p$ core levels, making the decomposition of the XMCD spectrum into the $L_{2}$ and $L_{3}$ components impossible. $.34,36,37 \mathrm{We}$ also note that the $M_{\text {orb }}$ of $\mathrm{Ti}$, which is obtained by integrating the entire XMCD spectrum, was below the detection limit $\left(\sim 0.1 \mu_{\mathrm{B}} /\right.$ atom $)$ of the present experiment. Considering that the magnetization is $45^{\circ}$ off from the X-ray incident direction, the obtained magnetic moments have been divided by $\cos 45^{\circ}=1 / \sqrt{2}$ in order to correct this effect. As shown in Fig. [6, the integral of the Fe XMCD spectrum has a large negative end value, suggesting that Fe has a large $M_{\text {orb }}$ parallel to the spin magnetic moment. The results of the sum rule yield $M_{\text {spin }}=2.45 \pm 0.16 \mu_{\mathrm{B}} / \mathrm{Fe}$ and $M_{\text {orb }}=0.59 \pm 0.08 \mu_{\mathrm{B}} / \mathrm{Fe}$. The obtained $M_{\text {spin }}$ is consistent with the values deduced from the first-principle calculations: $M_{\text {spin }}=2.45 \mu_{B} /$ Fe for $x=1 / 3$ and $M_{\text {spin }}=2.99 \mu_{B} /$ Fe for $x=1 . \underline{\underline{20}}$ The large $M_{\text {orb }}$ may be due to the degeneracy of the electron orbitals in the $\mathrm{Fe}^{2+}$ valence state and the partially filled $t_{2 g}$ level in a relatively weak crystal fields for the intercalated Fe atoms.

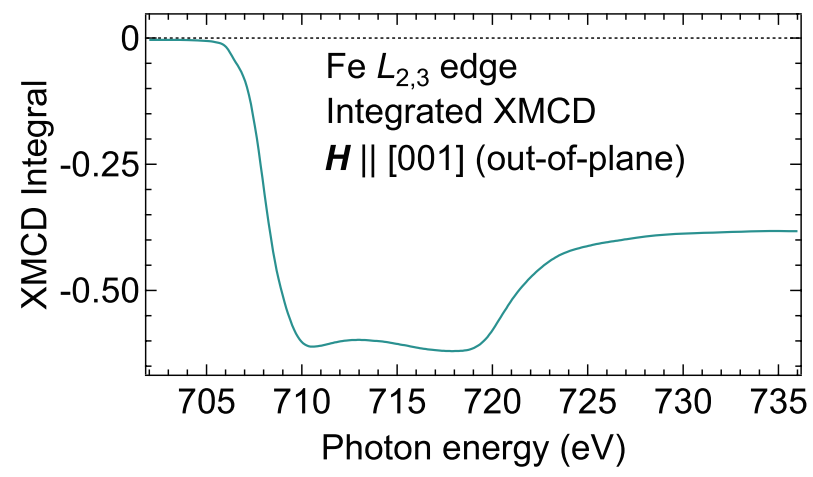

Figure 6: Integrated XMCD spectra of the $\mathrm{Fe}_{0.5} \mathrm{TiS}_{2}$ single crystal at the $\mathrm{Fe} L_{2,3}$ edge.

From the $M_{\text {spin }}$ and $M_{\text {orb }}$ values deduced by using the XMCD sum rule, one can deduce the uniaxial magnetic anisotropy energy $K_{\mathrm{u}}$ based on the Stoner-Wohlfarth model, $\underline{\underline{45}}$ in which one assumes uniaxial magnetic anisotropy and the coherent rotation of the magnetic domain. According to the model, the coercive field $H_{\mathrm{c}}$ is equal to $2 K_{\mathrm{u}} /\left(\mu_{0} M_{\mathrm{sat}}\right)$, where $M_{\text {sat }}=3.04 \pm 0.18 \mu_{\mathrm{B}} / \mathrm{Fe}$ is the saturation magnetization calculated as the sum of $M_{\text {spin }}$ and $M_{\text {orb }}$. From this relationship, the uniaxial MAE for the present $\mathrm{Fe}_{x} \mathrm{TiS}_{2}$ single crystal 
is estimated to be $K_{\mathrm{u}}=0.176 \pm 0.011 \mathrm{meV} / \mathrm{Fe}$ or $K_{\mathrm{u}}=0.244 \pm 0.015 \mathrm{MJ} / \mathrm{m}^{3}$ (note that $K_{\mathrm{u}}$ is defined positive for materials with PMA).

\section{Cluster-Model Calculation}

In order to explain the observed large $M_{\text {orb }}$ of Fe and the strong PMA of $\mu_{0} H_{\mathrm{c}} \simeq 1.0 \mathrm{~T}$ and $K_{\mathrm{u}}=0.244 \mathrm{MJ} / \mathrm{m}^{3}$, we calculated the XAS and XMCD spectra at the Fe $L_{2,3}$ edges using the CI cluster model. An $\left[\mathrm{FeS}_{6}\right]^{-10}$ cluster with weak trigonal distortion $\left(D_{3 d}\right.$ symmetry) was employed. In the present calculation, we first optimized the parameters $\Delta, U_{d d}, U_{d c}$, $(p d \sigma)$, and $10 D q$ in the $O_{h}$ symmetry (i.e., $\left.D_{\operatorname{trg}}=0\right)$ so that the calculated spectra well reproduce the experimental ones (for the definitions of $10 D q$ and $D_{\operatorname{trg}}$, see the inset of Fig. [7(b)) . The best-fit result was obtained with the parameter values listed in Table 1. The obtained parameter values are comparable to those deduced from the photoemission spectroscopy of $\mathrm{Fe}_{0.33} \mathrm{TiS}_{2} \cdot \underline{46}$ We then optimized the values of $H_{\text {mol }}$ and $D_{\operatorname{trg}}$ so that they reproduce the experimental $M_{\text {spin }}$ and $K_{\mathrm{u}}$, respectively. $K_{\mathrm{u}}$ has been calculated as the total energy difference between two spin configurations, $\boldsymbol{M}_{\text {spin }} \|$ [001] and $\boldsymbol{M}_{\text {spin }} \|$ [110]. Figure 7(a) shows the $H_{\text {mol }}$ dependence of the calculated spin magnetic moment $M_{\text {spin }}$. We note that modifying the values of $H_{\text {mol }}$ only resulted in the change in the XMCD intensity, without any variation in the characteristic spectral line shapes (not shown here). By comparing the calculated $M_{\text {spin }}$ with the experimental value, $H_{\text {mol }}$ has been estimated to be $5 \pm 1 \mathrm{meV}$. Figure 7 (b) shows the $D_{\text {trg }}$ dependence of the calculated magnetic anisotropy energy $K_{\mathrm{u}}$. It can be seen that the experimental $K_{\mathrm{u}}$ can be reproduced by only a slight change in $D_{\operatorname{trg}}$ of $\sim 1 \mathrm{meV}$. The calculated Fe $L_{2,3} \mathrm{XAS}$ and XMCD spectra are shown in Figs. 7(c) and 7(d) for $D_{\operatorname{trg}}=0 \mathrm{meV}$ and $D_{\operatorname{trg}}= \pm 1 \mathrm{meV}$. The calculated spectra well reproduce the experimental spectral features. It can also be seen that the spectral line shapes and the XMCD intensities are nearly insensitive to $D_{\operatorname{trg}}$, although such a small magnitude of $D_{\operatorname{trg}}$ can induce appreciable magnetic anisotropy as shown below. 
Table 1: Best-fit parameters for the configuration-interaction (CI) cluster-model calculations.

\begin{tabular}{ccccc}
\hline$\Delta$ & $U_{d d}$ & $U_{d c}$ & $(p d \sigma)$ & $10 D q$ \\
\hline $2.5 \mathrm{eV}$ & $5.0 \mathrm{eV}$ & $6.3 \mathrm{eV}$ & $-0.8 \mathrm{eV}$ & $0.7 \mathrm{eV}$ \\
\hline
\end{tabular}
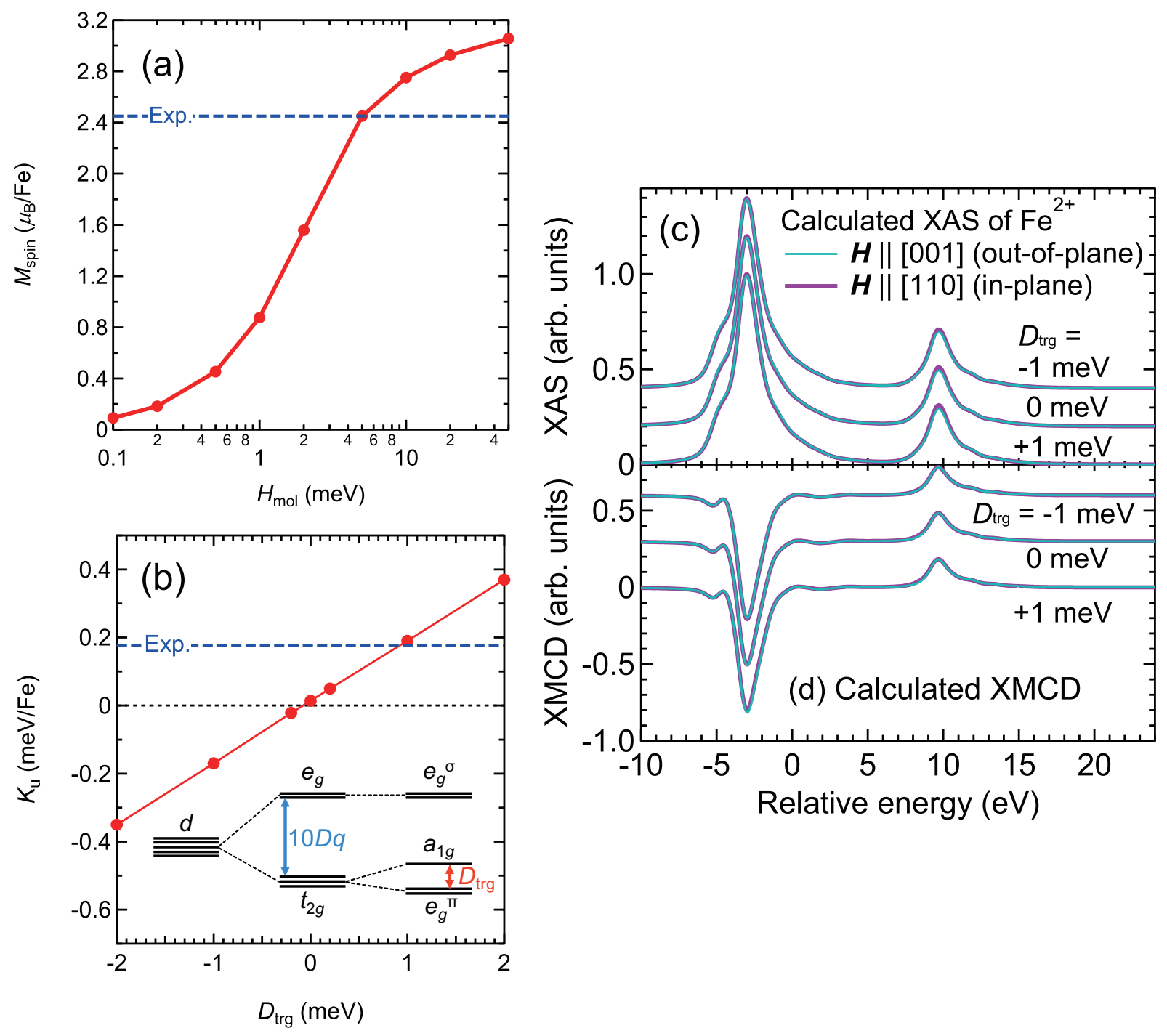

Figure 7: Results of CI cluster-model calculations at the Fe $L_{2,3}$ edge. (a) Molecular field $\left(H_{\text {mol }}\right)$ dependence of the spin magnetic moment per Fe atom $\left(M_{\text {spin }}\right)$. (b) Trigonal crystal field $\left(D_{\mathrm{trg}}\right)$ dependence of the magnetic anisotropy energy per Fe atom $\left(K_{\mathrm{u}}\right)$. In panels (a) and (b), blue dashed lines represent the experimental values. Inset of panel (b) depicts the crystal-field splitting in the case of $D_{\operatorname{trg}}>0$. (c),(d) Calculated XAS (c) and XMCD (d) spectra for $D_{\operatorname{trg}}=0 \mathrm{meV}$ and $D_{\operatorname{trg}}= \pm 1 \mathrm{meV}$. See Table 1 for the other parameter values used. 


\section{Discussion: Origin of the Strong PMA}

The microscopic origin of the strong PMA of Fe-intercalated TMDs is discussed by Parkin and Friend ${ }^{\underline{7}}$ based on the atomic multiplet model in an octahedral crystal field with a trigonal distortion. Here, we summarize their discussion and apply it to the present system. $\mathrm{An} \mathrm{Fe}^{2+}$ ion with $3 d^{6}$ electron configuration octahedrally coordinated by six anions has the ${ }^{5} T_{2 g}$ ground state, which is triply degenerate in the orbital part $\left(\left(t_{2 g}\right)^{4}\left(e_{g}\right)^{2}\right)$ and quintuply degenerate in the spin part $(S=2)$. (Note that the multielectron state ${ }^{5} T_{2 g}$ should be distinguished from the one-electron state $t_{2 g}$.) . The orbital triplet is isomorphic to the substates of orbital angular momentum $L=1$. In order to represent this orbital triplet, an effective orbital angular momentum operator $\boldsymbol{L}^{\mathrm{eff}}$ is introduced. Using $\boldsymbol{L}^{\mathrm{eff}}$, the Hamiltonian of the system can be written as follows:

$$
\hat{H}=-D_{\mathrm{trg}}\left(L_{z}^{\mathrm{eff}}\right)^{2}-\lambda \boldsymbol{L}^{\mathrm{eff}} \cdot \boldsymbol{S}+\boldsymbol{S} \cdot \boldsymbol{H}_{\mathrm{mol}}
$$

where the first, second, and third terms represent the energies of the trigonal crystal field $D_{\operatorname{trg}}(>0)$, SOI of the $3 d$ electrons $\lambda(>0)$, and the Zeeman effect by the molecular field $\boldsymbol{H}_{\text {mol }}$ (in the unit of measure of eV) originating from exchange interactions, respectively. Note that we have replaced the Zeeman term due to the external magnetic field $\mu_{B}\left(-\boldsymbol{L}^{\mathrm{eff}}+2 \boldsymbol{S}\right) \cdot \mu_{0} \boldsymbol{H}$, which is originally introduced by Parkin and Friend, $\underline{\underline{7}}$ by that due to the molecular field because we consider the FM state instead of the paramagnetic one. They have treated the first two terms as the primary terms and the Zeeman term as perturbation and have shown that the energy corrections for the out-of-plane and in-plane magnetic fields are of the first and second orders in $H_{\mathrm{mol}}$, respectively, resulting in the anisotropy of the $g$-factor. However, our cluster-model calculation shows that $\lambda(\simeq 50 \mathrm{meV})>H_{\mathrm{mol}}(\simeq 5 \mathrm{meV})>D_{\operatorname{trg}}(\simeq 1 \mathrm{meV})$.

We, therefore, treat the role of $D_{\operatorname{trg}}$ as perturbation to SOI and $H_{\text {mol }}$ in order to explain the magnetic anisotropy of $\mathrm{FM} \mathrm{Fe}_{x} \mathrm{TiS}_{2}$.

Figure 8 (a) schematically describes the energy diagram of an $\mathrm{Fe}^{2+}$ ion based on this 
model. The ${ }^{5} T_{2 g}$ ground state under the octahedral crystal field is split into three levels of $J^{\text {eff }}=1,2,3$ by SOI, where $\boldsymbol{J}^{\text {eff }}=\boldsymbol{L}^{\text {eff }}+\boldsymbol{S}$ is the effective total angular momentum. Since $\lambda$ is 1 order of magnitude larger than the thermal energy $k_{B} T(\sim 2.5 \mathrm{meV})$, only the $J^{\text {eff }}=1$ level has to be considered. The $J^{\text {eff }}=1$ level is then Zeeman-split into three sublevels of $m=1,0,-1$, where $m$ is the magnetic quantum number for $\boldsymbol{J}^{\text {eff }}$. The magnetic anisotropy energy can be deduced by calculating the difference of the perturbation energy of $D_{\operatorname{trg}}$ in the cases of out-of-plane $\left(\boldsymbol{H}_{\mathrm{mol}} \| \boldsymbol{z}\right)$ and in-plane molecular fields $\left(\boldsymbol{H}_{\mathrm{mol}} \| \boldsymbol{x}\right)$. The ground state $\left|J^{\mathrm{eff}}=1, m=1\right\rangle$ is expressed as follows in the cases of $\left(\boldsymbol{H}_{\mathrm{mol}} \| \boldsymbol{z}\right)$ and $\left(\boldsymbol{H}_{\mathrm{mol}} \| \boldsymbol{x}\right)$ :

$$
\begin{aligned}
\left|J^{\mathrm{eff}}=1, m=1\right\rangle_{z}= & \left|J^{\mathrm{eff}}=1, J_{z}^{\mathrm{eff}}=1\right\rangle \quad\left(\boldsymbol{H}_{\mathrm{mol}} \| \boldsymbol{z}\right), \\
\left|J^{\mathrm{eff}}=1, m=1\right\rangle_{x}= & \frac{1}{2}\left|J^{\mathrm{eff}}=1, J_{z}^{\mathrm{eff}}=1\right\rangle \\
& +\frac{1}{\sqrt{2}}\left|J^{\mathrm{eff}}=1, J_{z}^{\mathrm{eff}}=0\right\rangle \\
& \quad+\frac{1}{2}\left|J^{\mathrm{eff}}=1, J_{z}^{\mathrm{eff}}=-1\right\rangle \quad\left(\boldsymbol{H}_{\mathrm{mol}} \| \boldsymbol{x}\right),
\end{aligned}
$$

where the $\left|J^{\text {eff }}=1, J_{z}^{\text {eff }}\right\rangle$ terms can be written using the eigenstates of $L_{z}^{\text {eff }}$ and $S_{z}$ (denoted as $\left.\left|L_{z}, S_{z}\right\rangle\right)$ as

$$
\begin{aligned}
\left|J^{\mathrm{eff}}=1, \quad J_{z}^{\mathrm{eff}}=1\right\rangle & =\sqrt{\frac{6}{10}}|-1,2\rangle-\sqrt{\frac{3}{10}}|0,1\rangle+\sqrt{\frac{1}{10}}|1,0\rangle, \\
\left|J^{\mathrm{eff}}=1, \quad J_{z}^{\mathrm{eff}}=0\right\rangle & =\sqrt{\frac{3}{10}}|-1,1\rangle-\frac{2}{\sqrt{10}}|0,0\rangle+\sqrt{\frac{3}{10}}|1,-1\rangle, \\
\left|J^{\mathrm{eff}}=1, \quad J_{z}^{\mathrm{eff}}=-1\right\rangle & =\sqrt{\frac{1}{10}}|-1,0\rangle-\sqrt{\frac{3}{10}}|0,-1\rangle+\sqrt{\frac{6}{10}}|1,-2\rangle,
\end{aligned}
$$

Thus, the perturbation energies due to the trigonal crystal field $\left\langle-D_{\operatorname{trg}}\left(L_{z}^{\mathrm{eff}}\right)^{2}\right\rangle$ for the outof-plane and in-plane fields can be calculated as $-D_{\operatorname{trg}} / 4$ and $-D_{\operatorname{trg}} / 16$, respectively, to the first order of $D_{\text {trg }}$. This energy shift is schematically described in Fig. 8(b). This energy difference can be regarded as the origin of the PMA in the present system. We stress that this is a first-order perturbation effect, which is the reason why such a small magnitude of 
$D_{\operatorname{trg}}$ can give rise to the considerable magnitude of the magnetic anisotropy energy. The present result is consistent with the $D_{\operatorname{trg}}$ dependence of $K_{\mathrm{u}}$ shown in Fig. 7(b), in which $K_{\mathrm{u}}$ is nearly proportional to $D_{\text {trg }}$ when the crystal field is weak.

(a)

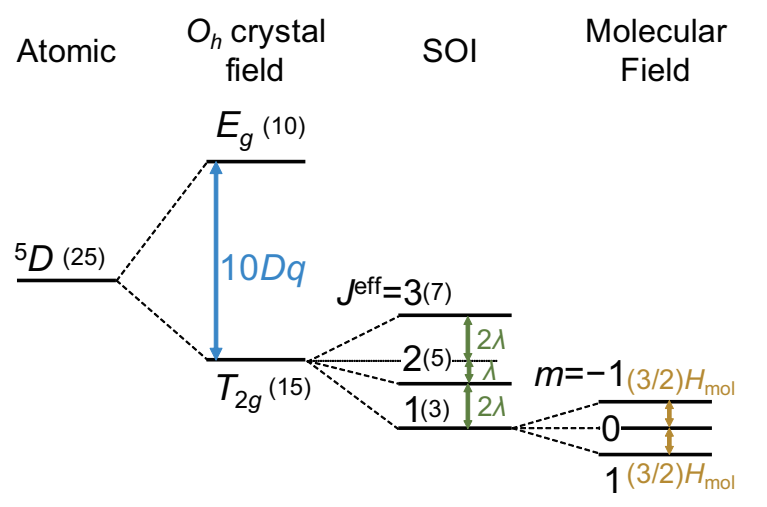

(b)

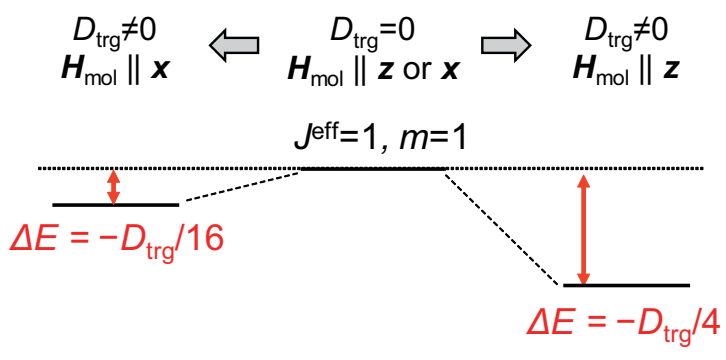

Figure 8: Schematic energy diagram of the $\mathrm{Fe}^{2+}$ ion $\left(3 d^{6}\right.$ electron configuration) octahedrally coordinated by six anions with small trigonal distortion. (a) Energy splitting due to the octahedral crystal field $(10 D q)$, SOI $(\lambda)$, and molecular field $\left(H_{\text {mol }}\right)$. The numbers in parentheses denote the degeneracy. (b) Energy shift due to the trigonal crystal field $D_{\operatorname{trg}}$ in the cases of out-of-plane $\left(\boldsymbol{H}_{\text {mol }} \| \boldsymbol{z}\right)$ and in-plane molecular fields $\left(\boldsymbol{H}_{\text {mol }} \| \boldsymbol{x}\right)$.

The present XMCD results show that there exists a large unquenched orbital moment of the intercalated Fe atom $\left(\simeq 0.59 \mu_{\mathrm{B}} / \mathrm{Fe}\right)$ in $\mathrm{Fe}_{0.5} \mathrm{TiS}_{2}$, which is considered to originate from the above-mentioned effective orbital angular momentum in the ${ }^{5} T_{2 g}$ state. The ratio of the orbital to spin angular momentum $\left(\left\langle L_{z}\right\rangle /\left\langle S_{z}\right\rangle=2 M_{\text {orb }} / M_{\text {spin }}\right)$ is calculated to be $0.48 \pm 0.07$. According to the previous XMCD measurements for $\mathrm{Fe}_{x} \mathrm{TiS}_{2}$ with $x \leq 0.33, \underline{27}$ the values of $\left\langle L_{z}\right\rangle /\left\langle S_{z}\right\rangle$ are deduced to be $0.49,0.48$, and 0.25 for $x=0.10,0.25$, and 0.33 , respectively. The $\left\langle L_{z}\right\rangle /\left\langle S_{z}\right\rangle$ value obtained for $x=0.5$ in the present study is out of this decreasing tendency with increasing $x$. Although the mechanism for this $x$ dependence of $\left\langle L_{z}\right\rangle /\left\langle S_{z}\right\rangle$ is not clear at present, hybridization effects between guest $\mathrm{Fe}$ and host $\mathrm{TiS}_{2}$, which have not been taken into account in the above discussion, might be relevant. We note that similar $x$ dependence has also been observed in the strength of the coercive field $H_{\mathrm{c}}$ of $\mathrm{Fe}_{x} \mathrm{TiS}_{2} \cdot \underline{\underline{9}} \cdot \underline{10}$ We expect that the complex $x$ dependence of $H_{\mathrm{c}}$ can be explained by the magnitude of the unquenched orbital magnetic moment of the intercalated $\mathrm{Fe}^{2+}$ ions. 


\section{Conclusion}

We have performed XAS and XMCD experiments on single crystals of heavily intercalated $\mathrm{Fe}_{x} \mathrm{TiS}_{2}(x \sim 0.5)$ in order to discuss the microscopic origin of the strong PMA and large $H_{\mathrm{c}}$ of this compound. We have confirmed that the grown single crystal showed a large $H_{\mathrm{c}}$ of $\mu_{0} H_{\mathrm{c}} \simeq 1.0 \mathrm{~T}$ and strong PMA with a magnetic anisotropy energy $K_{\mathrm{u}}$ of $0.244 \mathrm{MJ} / \mathrm{m}^{3}$.

From the XMCD result, the $\mathrm{Fe}^{2+}$ ions are shown to have a large orbital magnetic moment of $\simeq 0.59 \pm 0.08 \mu_{\mathrm{B}} / \mathrm{Fe}$. The strong magnetic anisotropy of $\mathrm{Fe}_{x} \mathrm{TiS}_{2}$ is attributed to the large orbital moment combined with the spin-orbit interaction and the trigonal crystal field.

\section{Acknowledgement}

A.F. and S.W.C. would like to thank D. D. Sarma for long-standing collaboration on a variety of interesting $3 d$ transition-metal compounds and for stimulating interaction during the last many decades. We thank Kenta Amemiya and Masako Sakamaki for valuable technical support at KEK-PF. This work was supported by Grants-in-Aid for Scientific Research from the JSPS (15H02109, 15K17696, 19K03741, and 20K14416). J.W.K. and S.W.C. were supported by the National Science Foundation (NSF) under Grant No. DMR-1629059. C.J.W. and S.W.C. partially were supported by the National Research Foundation of Korea (NRF) funded by the Ministry of Science and ICT(No. 2016K1A4A4A01922028 and No. 2020M3H4A2084417). Y.X.W. acknowledges support from Advanced Leading Graduate Course for Photon Science (ALPS) at the University of Tokyo. A.F. is an adjunct member of Center for Spintronics Research Network (CSRN), the University of Tokyo, under Spintronics Research Network of Japan (Spin-RNJ). The experiment was done under the approval of the Photon Factory Program Advisory Committee (Proposal No. 2016S2-005, No. 2016G066, and No. 2019G622).

The authors declare no competing financial interest. 


\section{References}

(1) Mahadevan, P.; Zunger, A.; Sarma, D. D. Unusual directional dependence of exchange energies in GaAs diluted with Mn: Is the RKKY description relevant? Phys. Rev. Lett. 2004, $\underline{93}, 177201$.

(2) Sarma, D. D.; Mahadevan, P.; Saha-Dasgupta, T.; Ray, S.; Kumar, A. Electronic structure of $\mathrm{Sr}_{2} \mathrm{FeMoO}_{6}$. Phys. Rev. Lett. 2000, 85, 2549-2552.

(3) Lee, J. Y.; Shin, J. H.; Lee, G. H.; Lee, C. H. Two-dimensional semiconductor optoelectronics based on van der Waals heterostructures. Nanomaterials (Basel) 2016, $\underline{6}$, 193.

(4) Choi, W.; Choudhary, N.; Han, G. H.; Park, J.; Akinwande, D.; Lee, Y. H. Recent development of two-dimensional transition metal dichalcogenides and their applications. Mater. Today 2017, 20, 116-130.

(5) Manzeli, S.; Ovchinnikov, D.; Pasquier, D.; Yazyev, O.; Kis, A. 2D transition metal dichalcogenides. Nat. Rev. Mater. 2017, 2, 17033.

(6) Pal, B.; Singh, A.; G., S.; Mahale, P.; Kumar, A.; Thirupathaiah, S.; Sezen, H.; Amati, M.; Gregoratti, L.; Waghmare, U. V. et al. Chemically exfoliated $\mathrm{MoS}_{2}$ layers: Spectroscopic evidence for the semiconducting nature of the dominant trigonal metastable phase. Phys. Rev. B 2017, 96, 195426.

(7) Parkin, S. S. P.; Friend, R. H. 3d transition-metal intercalates of the niobium and tantalum dichalcogenides. I. Magnetic properties. Philos. Mag. B 1980, 41, 65-93.

(8) Parkin, S. S. P.; Friend, R. H. 3d transition-metal intercalates of the niobium and tantalum dichalcogenides. II. Transport properties. Philos. Mag. B 1980, 41, 95-112.

(9) Inoue, M.; Matsumoto, M.; Negishi, H.; Sakai, H. Low field ac magnetic susceptibility 
measurements of intercalation compounds $\mathrm{M}_{x} \mathrm{TiS}_{2}(\mathrm{M}=3 \mathrm{~d}$ transition metals). J. Magn. Magn. Mater. 1985, $\underline{53}, 131-138$.

(10) Negishi, H.; Shoube, A.; Takahashi, H.; Ueda, Y.; Sasaki, M.; Inoue, M. Magnetic properties of intercalation compounds $\mathrm{M}_{x} \mathrm{TiS}_{2}(\mathrm{M}=3 \mathrm{~d}$ transition metal). $\underline{\mathrm{J} \text {. Magn. }}$

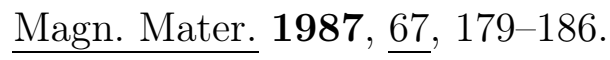

(11) Inoue, M.; Negishi, H. Interlayer spacing of 3d transition-metal intercalates of $1 \mathrm{~T}-\mathrm{CdI}_{2^{-}}$ type $\mathrm{TiS}_{2}$. J. Phys. Chem. 1986, 90, 235-238.

(12) Koyano, M.; Negishi, H.; Ueda, Y.; Sasaki, M.; Inoue, M. Electrical resistivity and thermoelectric power of intercalation compounds $\mathrm{M}_{x} \mathrm{TiS}_{2}(\mathrm{M}=\mathrm{Mn}, \mathrm{Fe}, \mathrm{Co}$, and $\mathrm{Ni})$.

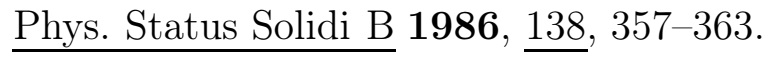

(13) Inoue, M.; Koyano, M.; Negishi, H.; Ueda, Y. Electronic properties of intercalation compound $\mathrm{Fe}_{x} \mathrm{TiS}_{2}$. J. Phys. Soc. Jpn. 1986, 55, 1400-1401.

(14) Negishi, H.; Koyano, M.; Inoue, M.; Sakakibara, T.; Goto, T. High field magnetization of $3 \mathrm{~d}$ transition metal intercalates $\mathrm{M}_{x} \mathrm{TiS}_{2}(\mathrm{M}=3 \mathrm{~d}$ metals). J. Magn. Magn. Mater. $1988, \underline{74}, 27-30$.

(15) Yoshioka, T.; Tazuke, Y. Magnetic properties of $\mathrm{Fe}_{x} \mathrm{TiS}_{2}$ system. J. Phys. Soc. Jpn. 1985, $\underline{54}, 2088-2091$.

(16) Negishi, H.; Ōhara, S.; Koyano, M.; Inoue, M.; Sakakibara, T.; Goto, T. Anisotropic spin-glass and cluster-glass of layered $\mathrm{Fe}_{x} \mathrm{TiS}_{2}$ crystals. J. Phys. Soc. Jpn. 1988, $\underline{57}$, 4083-4085.

(17) Choe, J.; Lee, K.; Huang, C.-L.; Trivedi, N.; Morosan, E. Magnetotransport in Feintercalated $T \mathrm{~S}_{2}$ : Comparison between $T=\mathrm{Ti}$ and Ta. Phys. Rev. B 2019, 99, 064420.

(18) Kuroiwa, Y.; Honda, H.; Noda, Y. Neutron magnetic scattering of intercalation compounds $\mathrm{Fe}_{x} \mathrm{TiS}_{2}$. Mol. Cryst. Liq. Cryst. Sci. Technol., Sect. A 2000, 341, 15-20. 
(19) Chiew, Y. L.; Miyata, M.; Koyano, M.; Oshima, Y. Ordering of intercalated Fe atoms in $\mathrm{Fe}_{x} \mathrm{TiS}_{2}$ structures clarified using transmission electron microscopy. J. Phys. Soc. Jpn. 2020, 89, 074601.

(20) Suzuki, N.; Yamasaki, T.; Motizuki, K. Electronic band structures of intercalation compounds of 3d transition metals with $\mathrm{TiS}_{2}$. J. Magn. Magn. Mater. 1987, 70, 64-66.

(21) Suzuki, N.; Yamasaki, T.; Motizuki, K. Electronic band structures and bond orders of $\mathrm{M}_{1 / 3} \mathrm{TiS}_{2}(\mathrm{M}=\mathrm{Mn}, \mathrm{Fe}, \mathrm{Co}, \mathrm{Ni})$. J. Phys. Soc. Jpn. 1989, 58, 3280-3289.

(22) Martinez, H.; Tison, Y.; Baraille, I.; Loudet, M.; Gonbeau, D. Experimental (XPS/STM) and theoretical (FLAPW) studies of model systems $\mathrm{M}_{1 / 4} \mathrm{TiS}_{2}(\mathrm{M}=\mathrm{Fe}$, Co, Ni): influence of the inserted metal. J. Electron. Spectrosc. Relat. Phenom. 2002, $\underline{125}, 181-196$.

(23) Ueda, Y.; Negishi, H.; Koyano, M.; Inoue, M.; Soda, K.; Sakamoto, H.; Suga, S. Resonant photoemission studies of $3 d$ transition metal intercalates of $\mathrm{TiS}_{2}$. Solid State Commun. 1986, 57, 839-842.

(24) Ueda, Y.; Fukushima, K.; Negishi, H.; Inoue, M.; Taniguchi, M.; Suga, S. Photoemission studies on intercalation compounds of $\mathrm{M}_{x} \mathrm{TiS}_{2}(\mathrm{M}=3 \mathrm{~d}$ transition metals). $\underline{\mathrm{J} \text {. Phys. Soc. }}$ Jpn. 1987, 56, 2471-2476.

(25) Fujimori, A.; Suga, S.; Negishi, H.; Inoue, M. X-ray photoemission and Auger-electron spectroscopic study of the electronic structure of intercalation compounds $M_{x} \mathrm{TiS}_{2}$ $(\mathrm{M}=\mathrm{Mn}, \mathrm{Fe}, \mathrm{Co}$, and Ni). Phys. Rev. B 1988, $\underline{38}, 3676-3689$.

(26) Suga, S. Angle-resolved, resonance-and inverse-photoemission studies of transition metal intercalated $\mathrm{TiS}_{2}$. Mol. Cryst. Liq. Cryst. Sci. Technol., Sect. A 2000, 341, 9-14.

(27) Yamasaki, A.; Imada, S.; Sekiyama, A.; Suga, S.; Matsushita, T.; Muro, T.; Saitoh, Y.; 
Negishi, H.; Sasaki, M. Angle-resolved photoemission spectroscopy and magnetic circular dichroism in Fe-intercalated $\mathrm{TiS}_{2}$. Surf. Rev. Lett. 2002, 9, 961-966.

(28) Suga, S.; Tusche, C.; ichiro Matsushita, Y.; Ellguth, M.; Irizawa, A.; Kirschner, J. Momentum microscopy of the layered semiconductor $\mathrm{TiS}_{2}$ and $\mathrm{Ni}$ intercalated $\mathrm{Ni}_{1 / 3} \mathrm{TiS}_{2}$. New J. Phys. 2015, 17, 083010.

(29) Tazuke, Y.; Ohta, Y.; Miyamoto, S. Exchange interactions in $\mathrm{Fe}_{x} \mathrm{TiS}_{2}$. J. Phys. Soc. Jpn. 2005, 74, 2644-2645.

(30) Tazuke, Y.; Miyashita, T.; Nakano, H.; Sasaki, R. Magnetic properties of $\mathrm{M}_{x} \mathrm{TiSe}_{2}$ (M=Mn, Fe, Co). Phys. Status Solidi C 2006, 3, 2787-2790.

(31) Lévy, F., Ed. Intercalated Layered Materials; D. Reidel Publishing Company: Dordrecht, Holland, 1979.

(32) Furuse, M.; Okano, M.; Fuchino, S.; Uchida, A.; Fujihira, J.; Fujihira, S.; Kadono, T.; Fujimori, A.; Koide, T. HTS vector magnet for magnetic circular dichroism measurement. IEEE Trans. Appl. Supercond. 2013, 23, 4100704.

(33) Shibata, G.; Kitamura, M.; Minohara, M.; Yoshimatsu, K.; Kadono, T.; Ishigami, K.; Harano, T.; Takahashi, Y.; Sakamoto, S.; Nonaka, Y. et al. Anisotropic spin-density distribution and magnetic anisotropy of strained $\mathrm{La}_{1-x} \mathrm{Sr}_{x} \mathrm{MnO}_{3}$ thin films: angledependent x-ray magnetic circular dichroism. npj Quantum Mater. 2018, $\underline{3}, 3$.

(34) Carra, P.; Thole, B. T.; Altarelli, M.; Wang, X. X-ray circular dichroism and local magnetic fields. Phys. Rev. Lett. 1993, 70, 694-697.

(35) Thole, B. T.; Carra, P.; Sette, F.; van der Laan, G. X-ray circular dichroism as a probe of orbital magnetization. Phys. Rev. Lett. 1992, $\underline{68}$, 1943-1946.

(36) Teramura, Y.; Tanaka, A.; Jo, T. Effect of Coulomb interaction on the x-ray magnetic 
circular dichroism spin sum rule in $3 d$ transition elements. J. Phys. Soc. Jpn. 1996, $\underline{65}$, $1053-1055$.

(37) Piamonteze, C.; Miedema, P.; de Groot, F. M. F. Accuracy of the spin sum rule in XMCD for the transition-metal $L$ edges from manganese to copper. Phys. Rev. B 2009, $\underline{80}, 184410$.

(38) Stöhr, J.; König, H. Determination of spin- and orbital-moment anisotropies in transition metals by angle-dependent x-ray magnetic circular dichroism. Phys. Rev. Lett. $1995, \underline{75}, 3748-3751$.

(39) Dürr, H. A.; van der Laan, G. Magnetic circular x-ray dichroism in transverse geometry: Importance of noncollinear ground state moments. Phys. Rev. B 1996, 54, R760-R763.

(40) Tanaka, A.; Jo, T. Resonant $3 d, 3 p$ and $3 s$ photoemission in transition metal oxides predicted at $2 p$ threshold. J. Phys. Soc. Jpn. 1994, $\underline{63}$, 2788-2807.

(41) Regan, T. J.; Ohldag, H.; Stamm, C.; Nolting, F.; Lüning, J.; Stöhr, J.; White, R. L. Chemical effects at metal/oxide interfaces studied by x-ray-absorption spectroscopy. Phys. Rev. B 2001, $\underline{64}, 214422$.

(42) Chen, C. T.; Idzerda, Y. U.; Lin, H.-J.; Smith, N. V.; Meigs, G.; Chaban, E.; Ho, G. H.; Pellegrin, E.; Sette, F. Experimental confirmation of the x-ray magnetic circular dichroism sum rules for iron and cobalt. Phys. Rev. Lett. 1995, $\underline{75}, 152-155$.

(43) Kimura, A.; Suga, S.; Matsushita, T.; Imada, S.; Shino, N.; Saitoh, Y.; Shigeoka, H.; Daimon, H.; Kinoshita, T.; Kakizaki, A. et al. Electronic structure of Ni intercalated $\mathrm{TiS}_{2}$ probed by angle resolved and $2 p$ core resonance photoemission as well as by $2 p$ core absorption spectroscopy. Jpn. J. Appl. Phys. 1993, $\underline{32}$, 255-257.

(44) Cao, Y.; Liu, X.; Kareev, M.; Choudhury, D.; Middey, S.; Meyers, D.; Kim, J.- 
W.; Ryan, P.; Freeland, J.; Chakhalian, J. Engineered Mott ground state in a $\mathrm{LaTiO}_{3+\delta} / \mathrm{LaNiO}_{3}$ heterostructure. Nat. Commun. 2016, $\underline{7}, 10418$.

(45) Stoner, E. C.; Wohlfarth, E. P. A mechanism of magnetic hysteresis in heterogeneous alloys. Philos. Trans. R. Soc., A 1948, 240, 599-642.

(46) Bocquet, A. E.; Mizokawa, T.; Saitoh, T.; Namatame, H.; Fujimori, A. Electronic structure of $3 d$-transition-metal compounds by analysis of the $2 p$ core-level photoemission spectra. Phys. Rev. B 1992, 46, 3771-3784. 